\title{
Elbow Torque Estimated using a Nonlinear Observer
}

\author{
Emer P. Doheny, Madeleine M. Lowery * and Annraoi de Paor
}

School of Electrical and Electronic Engineering, University College Dublin, Dublin 4, Ireland

\begin{abstract}
An inverse dynamics model has been designed, using control theory, to estimate elbow joint torque per unit moment of inertia based on a measured movement trajectory. The physiological parameters required by the model (undamped natural frequency and damping ratio of the elbow joint) were measured experimentally using the pendulum test at the elbow joint. Eight subjects participated in the experimental study, which included ten iterations of the standard pendulum test at the elbow joint, as well as ten iterations of the pendulum test with an additional known weight strapped to the wrist. Electrogoniometry was used to record the movement of the forearm during the tests, and surface electromyography of the elbow flexor and extensor muscles was used to ensure that the movements were purely passive. An equation was then derived to calculate the moment of inertia of the forearm as it rotated about the elbow joint. The inverse dynamics model developed in this study could be a useful tool for clinicians in analysing kinematics in patients with neuromuscular or orthopaedic disease.
\end{abstract}

Keywords: Inverse dynamics; Moment of inertia; Model; Pendulum test.

\section{Introduction}

Inverse dynamics models are commonly used to predict joint torque based on measurements of joint position. Inverse dynamics has been used as a noninvasive, convenient method to improve the sensitivity of clinical motion analysis in patients with cerebellar lesions [15], Parkinson's disease, spasticity and orthopaedic injury $[7,8]$. It has also been used to improve adaptive control of neuroprostheses [16]. Furthermore, inverse dynamics could be used in the development of a dynamic neuromusculoskeletal model, to examine the neuromuscular or biomechanical effects of a change in joint geometry following total joint replacement. Provided accurate values for the moment of inertia, mass of body segments, position of the centre of gravity, undamped natural frequency and damping ratio of the joint are known, reliable joint torque predictions may be made. However, these values are not easy to obtain and reported values vary considerably [17].

An inverse dynamics model was developed in this study, using control theory, to track the net joint torque developed during elbow flexion and extension based on the movement trajectory of the forearm about the elbow. An experimental study was performed, which allowed the moment of inertia, undamped natural frequency and the damping ratio of the elbow joint to be calculated easily for individual subjects.

\section{Model}

The aim of this model was to estimate the net flexor torque per unit moment of inertia, $\frac{T_{f}}{I}$, based on the measured movement trajectory, $\varphi_{h}(t)$, as illustrated in 
Figure 1.

$$
\stackrel{T_{f}}{I}(s) \longrightarrow G(s), h\left(\phi_{h}\right) \longrightarrow \phi_{h}(s)
$$

Fig. 1: Transfer function representation of the modelled system. $\frac{T_{f}}{I}(s)$ is the Laplace transform of the net elbow flexor torque per unit moment of inertia, $\varphi_{h}(s)$ is the movement trajectory, $G(s)$ represents the linear part of the process, $h\left(\varphi_{h}\right)$ is a nonlinear feedback function.

To address this problem, the dynamics of the elbow joint were first examined - the process model in a control systems approach. An observer was then designed, based on the process model, and finally a controller was developed. The resulting system should predict an accurate replica of the unobservable signal $\frac{T_{f}}{I}(t)$ based on the measured joint movement, $\varphi_{h}^{I}(t)$. In this model, the upper arm was considered to be vertical, and $\varphi_{h}$ was defined as the angle of the forearm relative to the horizontal.

\subsection{The Dynamics of the Elbow Joint}

A representation of the forearm rotating about the elbow joint is presented in Figure 2. Assuming viscous friction, the equation of motion of the forearm is:

$I \frac{d^{2} \varphi_{h}}{d t^{2}}=-F \frac{d \varphi_{h}}{d t}-m g l \cos \varphi_{h}+T_{f}$

where $I$ is the moment of inertia of the forearm, $F$ is the coefficient of viscous friction, $m$ is the mass of the forearm, $g$ is the acceleration due to gravity (taken as $9.81 \mathrm{~m} / \mathrm{s}^{2}$ ), $l$ is the distance of the centre of gravity of the forearm from the centre of the elbow joint and $T_{f}$ is the net elbow flexor torque.

By introducing the standard [3] dynamical indices of damping ratio $\zeta$ and undamped natural frequency $\omega_{n}$, equation 1 may be converted to the form

$$
\frac{d^{2} \varphi_{h}}{d t^{2}}+2 \zeta \omega_{n} \frac{d \varphi_{h}}{d t}+\omega_{n}^{2} \cos \varphi_{h}=\frac{T_{f}}{I}
$$

where $\omega_{n}$ is the undamped natural frequency of the elbow joint, and $\zeta$ is the damping ratio of the elbow joint. These dynamical indices are defined by:

$$
\begin{aligned}
& \omega_{n}=\sqrt{\frac{m g l}{I}} \\
& \zeta=\frac{F}{2 \sqrt{m g l I}}
\end{aligned}
$$

By adding $\omega_{n}^{2} \varphi_{h}$ to both sides of equation 2, it may be rewritten as:

$\frac{d^{2} \varphi_{h}}{d t^{2}}+2 \zeta \omega_{n} \frac{d \varphi_{h}}{d t}+\omega_{n}^{2} \varphi_{h}=\frac{T_{f}}{I}-h\left(\varphi_{h}\right)$

where the nonlinear feedback function, $h\left(\varphi_{h}\right)$, is defined in equation 6.

$h\left(\varphi_{h}\right)=\omega_{n}^{2}\left(\cos \varphi_{h}-\varphi_{h}\right)$

By taking the Laplace transform of equation 5, subject to zero state initial conditions, $\varphi_{h}(0)=0$ and $\frac{d \varphi_{h}}{d t}(0)=0$, the process may be represented by equation 7, and Figure 3.

$\varphi_{h}(s)=G(s)\left(\frac{T_{f}}{I}(s)-\mathcal{L} h\left(\varphi_{h}\right)\right)$

where $\mathcal{L}$ denotes Laplace Transform. The linear part of the process, $G(s)$, is then characterised by the transfer function given in equation 8 :

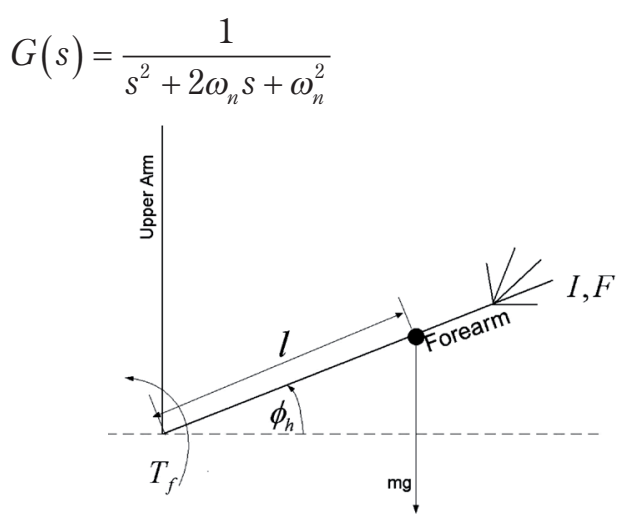

Fig. 2: Representation of the forearm rotating about the elbow.

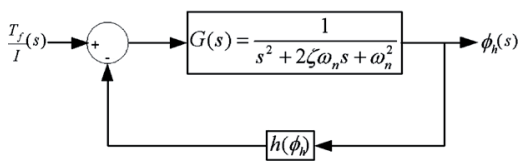

Fig. 3: The nonlinear process considered. $G(s)$ represents the linear part of the process, and $h\left(\varphi_{h}\right)$ is a nonlinear feedback function.

\subsection{Nonlinear Observer Design}

A nonlinear observer was constructed, Figure 4 , driven by the measured $\varphi_{h}(t)$, with nonlinear feedback function $h\left(\varphi_{h}\right)$ an accurate model of the linear part of the process, $G(s)$, and an appropriately tuned controller, $C(s)$. The state variables used in a Simulink implementation of the model are indicated using $x_{1}$ to $x_{4}$. 


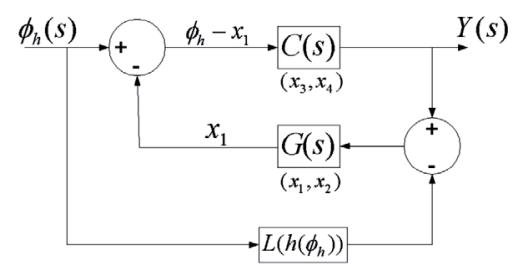

Fig. 4: The structure of the nonlinear observer. $\phi_{h}$ is the elbow angle relative to the horizontal, $Y$ is the predicted value of $\frac{T_{f}}{I}, C(s)$ is the controller, $G(s)$ is the linear process model, $h\left(\phi_{h}\right)$ is the nonlinear feedback function, $x_{1}, x_{2}, x_{3}$ and $x_{4}$ are state variables.

The following equation applies to the zero-state response:

$$
\begin{aligned}
& Y(s)=C(s)\left[\varphi_{h}(s)-G(s)\left(Y(s)-\mathcal{L} h\left(\varphi_{h}\right)\right)\right](9) \\
& \mathcal{L}\left(x_{1}\right)=X_{1}(s)=G(s)\left(Y(s)-\mathcal{L} h\left(\varphi_{h}\right)\right)
\end{aligned}
$$

Comparing equations 10 and 7 it is clear that if $Y(s) \approx \frac{T_{f}}{I}(s)$, then $x_{1}(t) \approx \varphi_{h}(t)$. Substituting equation 7 into equation 9 leads to:

$$
Y(s)=\left(\frac{C(s) G(s)}{1+C(s) G(s)}\right) \frac{T_{f}}{I}(s)
$$

Equation 11 represents the linear feedback system shown in Figure 5. It is clear from this that, provided $C(s)$ is a good controller for $G(s)$, the output signal $Y(s)$ will track the input signal $\frac{T_{f}}{I}(s)$ with small error and so, in the time domain, the generated observer output signal $y(t)$ will be an accurate replica of the unknown input $\frac{T_{f}}{I}(t)$.

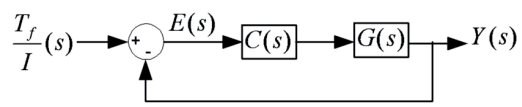

Fig. 5: Equivalent linear feedback system relating $Y(s)$ to $\frac{T_{f}}{I}(s)$. $E(s)$ is the error signal.

\subsection{Controller Design}

Initially, a controller of the form in equation 12 was chosen.

$C(s)=\frac{k\left(s^{2}+2 \zeta \omega_{n} s+\omega_{n}^{2}\right)}{s}$

which gave the forward path transfer function

$$
C(s) G(s)=\frac{k}{s}
$$

Combining equations 13 and 11 leads to:

$$
Y(s)=\frac{1}{1+\frac{s}{k}} \frac{T_{f}}{I}(s)
$$

Equation 14 represents $y(t)$ tracking $\frac{T_{f}}{I}(t)$ through a first order low-pass filter of time constant $k$ seconds i.e., of $3 \mathrm{~dB}$ bandwidth $k$ radians per second. Provided that $k$ is chosen so that this bandwidth accommodates all significant frequency components of $\frac{T_{f}}{I}$, then the generated observer output $y(t)$ will be a close match to the unmeasurable process input $\frac{T_{f}}{I}(t)$.

The controller transfer function in equation 12 can be rearranged as

$C(s)=2 k \zeta \omega_{n}\left(1+\frac{1}{s 2 \frac{\zeta}{\omega_{n}}}+s \frac{1}{2 \zeta \omega_{n}}\right)$

which is of the form

$C(s)=2 k \zeta \omega_{n}\left(1+\frac{1}{s T_{i}}+s T_{d}\right)$

Equation 16 is the classical proportional, integral plus derivative (PID) controller, with proportional gain $2 k \zeta \omega_{n}$, integral action time $T_{i}=\frac{2 \zeta}{\omega}$ and derivative action time $T_{d}=\frac{1}{2 \zeta \omega_{n}}$ [13]. Equation 16 cannot be implemented exactly in state variable form. It is necessary to approximate the derivative channel transfer function using the approximation $s T_{d}=\frac{s T_{d}}{1+a T_{d} s}$, where $a<<1$. This represents pure derivative action passed through a first order low-pass filter with time constant $a T_{d}$. It can be implemented by a gain in cascade with an integrator in a feedback loop, as shown in Figure 6, through the following simple manipulation:

$\frac{s T_{d}}{1+a T_{d} s}=\frac{1}{a}\left(\frac{1}{1+\frac{1}{a T_{d}} \frac{1}{s}}\right)$

The block diagram representation of the modified controller is shown in Figure 7. The state variable equations describing this are:

$\frac{d x_{3}}{d t}=k \omega_{n}^{2}\left(\varphi_{h}-x_{1}\right)$ 
$\frac{d x_{4}}{d t}=\frac{2 \zeta \omega_{n}}{a}\left(\frac{2 k \zeta \omega_{n}}{a}\left(\varphi_{h}-x_{1}\right)-x_{4}\right)$

with output

$$
y=2 k \zeta \omega_{n}\left(\varphi_{h}-x_{1}\right)+x_{3}+\frac{2 \zeta \omega_{n}}{a}\left(\varphi_{h}-x_{1}\right)-x_{4}
$$

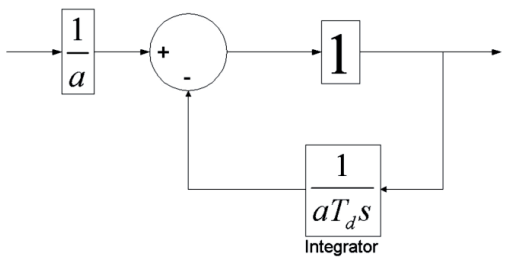

Fig. 6: The manipulation applied to the derivative channel is shown here. This is necessary in order to implement the controller in Simulink.

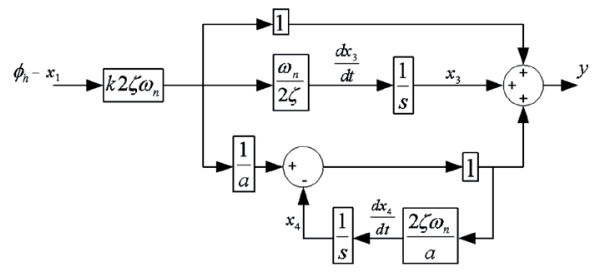

Fig. 7: Block diagram representation of the modified PID controller

The state variable equations for the process model are

$\frac{d x_{1}}{d t}=x_{2}$

$\frac{d x_{2}}{d t}=-\omega_{n}^{2} x_{1}-2 \zeta \omega_{n} x_{2}+y-h\left(\varphi_{h}\right)$

with $h\left(\varphi_{h}\right)$ defined by equation 6 .

In the process model state variable equations 22 and $23, x_{1}$ is the counterpart of $\varphi_{h}$ and $x_{2}$ of $\frac{d \varphi_{h}}{d t}$. Hence, these were initialised with $x_{1}=0$ and $x_{2}=0$. It is noted from Figure 7 that if $\varphi_{h}(0)=0, x_{1}(0)=0$ and if $x_{4}(0)=0$, then $y(0)=x_{3}(0)$. However, it is readily seen from equation 2 that if forearm motion starts from rest, $\frac{T_{f}}{I}(0)=h(0)=\omega_{n}^{2}$. Therefore, $x_{3}$ and $x_{4}$ were initialised with $x_{3}=\omega_{n}^{2}$ and $x_{4}=0$.

The controller parameters $k$ and a were tuned in this model as follows: $k$ was tuned by increasing it until two successive traces of $y(t)$ showed no significant change. In the control theory literature, the parameter a is conventionally taken as $a<<0.1$. It was found here in preliminary simulation experiments that the value $a=0.006$ allowed very close tracking of $\frac{T_{f}}{I}$ by $y$.

\subsection{Experimental Procedure}

The values of $\zeta, \omega_{n}$ and $I$ were calculated experimentally, using the pendulum test at the elbow joint. The pendulum test, developed initially for the knee [18], is a commonly used diagnostic test of spasticity [4], [11], [14], and has also been used to measure biomechanical parameters [5] and investigate their variation in different patient populations [9], or to quantify muscle tone [10]. In this study, the pendulum test was used to estimate the damping ratio, $\zeta$, the undamped natural frequency, $\omega_{n}$, and the moment of inertia, $I$, of the human elbow joint for use in modelling elbow joint kinematics. A similar added mass idea, resulting in a completely equivalent result, but derived and formulated independently here, was used in [12] to measure the moment of inertia of the lower leg rotating about the knee joint.

\subsection{Equation of Motion}

During a pendulum test, the equation of motion differs to that of the modelled system. Firstly, the movement should be passive, hence there is no muscle torque. Secondly, the forearm moves in the vertical plane rather than the horizontal. The resulting equation of motion is given in equation 24 , where $\varphi_{v}$ represents the angle of the forearm relative to the vertical.

$I \frac{d^{2} \varphi_{v}}{d t^{2}}=-F \frac{d \varphi_{v}}{d t}-m g l \sin \varphi_{v}$

The system can be linearised, by assuming that $\varphi_{v}$ does not exceed $1 \mathrm{rad}$, hence $\varphi_{v} \gg \sin \varphi_{v}$ The linearised equation of motion is given in equation 25

$I \frac{d^{2} \varphi_{v}}{d t^{2}}=-F \frac{d \varphi_{v}}{d t}-m g l \varphi_{v}$

which is equivalent to:

$\frac{d^{2} \varphi_{v}}{d t^{2}}+2 \zeta \omega_{n} \frac{d \varphi_{v}}{d t}+\omega_{n}^{2} \varphi_{v}=0$

The solution of this equation of motion for $-1<\varphi_{v}<1$ rad and $\frac{d \varphi_{\nu}}{d t}(0)=0$, is given by equation 27.

The derivative of equation 27 leads to the expression given in equation 28. 


$$
\begin{aligned}
& \varphi_{v}(t)=\varphi_{v}(0) e^{-\zeta \omega_{n} t}\left(\frac{\zeta}{\sqrt{1-\zeta^{2}}} \sin \left(\omega_{n} \sqrt{1-\zeta^{2}} t\right)+\cos \left(\omega_{n} \sqrt{1-\zeta^{2}} t\right)\right) \\
& \frac{d \varphi_{v}}{d t}=-\varphi_{v}(0) e^{-\zeta \omega_{n} t} \frac{\omega_{n}}{\sqrt{1-\zeta^{2}}} \sin \left(\omega_{n} \sqrt{1-\zeta^{2}} t\right)
\end{aligned}
$$

The turning points, which correspond to $\frac{d \varphi_{\nu}}{d t}=0$, are given by the zeros of the sine function:

$$
\omega_{n} \sqrt{1-\zeta^{2}} t=m \pi \quad m=0,1,2,3, \ldots
$$

The solution $\varphi_{v}(t)$ is presented in Figure 8 , with turning points for the various values of $\mathrm{m}$ indicated. The peak values $\varphi_{v p 1}, \varphi_{v p 2}, \varphi_{v p 3}$ etc., correspond to even values of $\mathrm{m}, \mathrm{m}=0,2,4,6, \ldots$

From equation 27, an expression for the logarithmic decrement, $\delta$, may by deduced, see equation 30 . $\delta$ could also be defined using successive trough values.

$$
\delta=\ln \frac{\varphi_{v p 1}}{\varphi_{v p 2}}=\ln \frac{\varphi_{v p 2}}{\varphi_{v p 3}}=\ln \frac{\varphi_{v p 3}}{\varphi_{v p 4}}=\ldots=\frac{2 \pi \zeta}{\sqrt{1-\zeta^{2}}}
$$

(i)

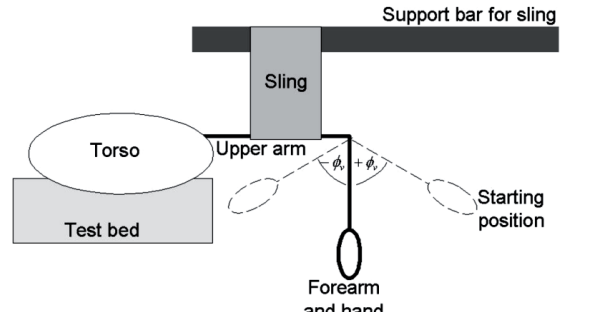

(ii)

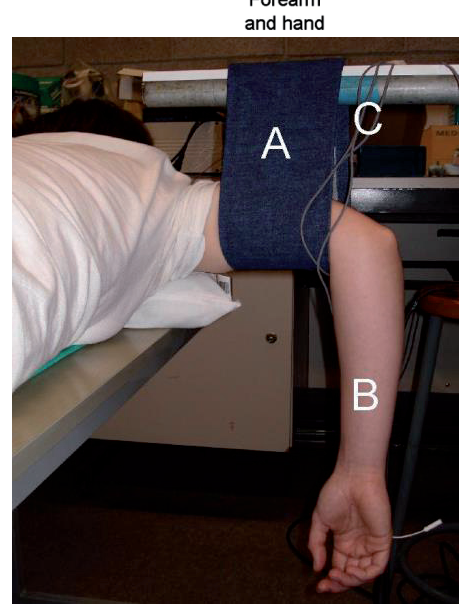

Fig. 8: The experimental set-up used during the pendulum tests. $i$ : A cross-section through the subject's chest is illustrated. The angle of the forearm relative to the vertical, $\varphi_{\nu}$, is shown for forearm swings in both directions. ii: A: The secure inelastic sling used to support the upper arm. B: The forearm hanging in its final position. $C$ : Cables transmitting surface EMG and electrogoniometer data to an analog-digital converter for analysis.

\subsection{Experimental Procedure}

Eight healthy subjects (4 Male, 4 Female; weight: 58- $90 \mathrm{~kg}$ ) took part in this study. The movement trajectory of the forearm was measured using an electrogoniometer, while surface EMG was recorded to ensure that the movement was passive. An electrogoniometer (Biometrics Ltd.) was attached using medical tape along the lateral side of the right elbow. The subject was asked to flex and extend their elbow between $\varphi_{v}=-1 \mathrm{rad}$ and $\varphi_{v}=1 \mathrm{rad}$ while the movement was recorded, in order to confirm that all joint angles could be captured. Surface EMG was recorded from the biceps, brachioradialis and triceps muscles using the Delsys Bagnoli 8 system (bandpass filter: 20 - $450 \mathrm{~Hz}$ ). Skin was prepared by gentle abrasion and cleansing with alcohol. Electrodes were located according to the SENIAM [6] recommended locations for each muscle, with a fixed inter-electrode distance of $10 \mathrm{~mm}$. EMG data was sampled at $1250 \mathrm{~Hz}$, and electrogoniometer data at $250 \mathrm{~Hz}$. Signals were then stored on a PC after A-D conversion (CED 1401), and analysed using Matlab (the Mathworks). The subject lay on the test bed in the prone position, and with their head facing to the left. Their right upper arm was held horizontally by a strong custom designed sling, which was supported by a horizontal bar. The right shoulder was relaxed and abducted $90^{\circ}$ from the torso, so that the forearm hung vertically and swung freely if disturbed. The subject was encouraged to relax, and not to assist or resist the pendulum motion. The surface EMG recordings were monitored during the experiments to ensure that no active muscle force was developed. If EMG activity was detected during the pendulum tests, those recordings were rejected and repeated.

The forearm was lifted to an angle less than 1 radian using a piece of string, which was tied loosely around the wrist, and released suddenly, so that the natural swing of the forearm was not interfered with. After the forearm swinging motion had stopped completely, the procedure was repeated a further nine times. The entire procedure was then repeated with a $0.45 \mathrm{~kg}$ wrist weight (Nike,) securely 
fastened around the subject's wrist, these tests were used to calculate the moment of inertia below. The experimental set-up is shown in Figure 8.

\subsection{Data Analysis}

A typical pendulum test output is shown in Figure 9. All time points where $\varphi_{v}=0$ were first identified, and using these data points, the peaks and troughs of the curve were calculated by finding the maximum and minimum values respectively between these time points. $\varphi_{v p 1}$ corresponded to the first peak, $\varphi_{v p 2}$ corresponded to the second peak, and successive peaks were referred to using the same notation. Troughs were identified in the same manner, as $\varphi_{v t 1,}$ $\varphi_{v t 2}$, as illustrated in Figure 8. The period of the signal, $T$, was then calculated by finding the time between successive peaks, or troughs. The methods used to calculate $\zeta$, wn and $I$ are described below, and the calculated values for each subject, as well as mean values, are presented in Table I. An inverse dynamics model simulation for subject 5 is presented in figure 10.

1) Initial Estimate of the Damping Ratio of the Elbow Joint, $\zeta$ : The value of the logarithmic decrement, $\delta$, was determined using equation 30 . Ideally, the value of $\delta$ should be the same, irrespective of the chosen peaks or troughs in equation 30, however due to variability in the experimental conditions this is not always true. Hence, for each pendulum test, $\delta$ was calculated using eight different pairs of peaks and troughs, and that pendulum test was only considered valid if the standard deviation between the $\delta$ values was less than 30\% of the mean of those values. Ten pendulum tests were conducted, with and without the added weight, the mean of the $\delta$ values for each valid pendulum test was used in all subsequent calculations. An initial estimate of $\delta$, with and without an added weight at the wrist, was calculated using equation 30 .

Table 1: The undamped natural frequency, $\omega_{n}$, damping ratio, $\zeta$, moment of inertia, $I$, and friction coefficient, $F$, of the elbow joint for each subject. $\omega_{n a}$ and $\zeta_{a}$ are the undamped natural frequency and damping ratio respectively, with an additional weight at the wrist. The mean values which were used in the model are presented. Reported values are correct to two decimal places.

\begin{tabular}{|l|l|l|l|l|l|l|l|l|l|}
\hline Subject & Gender & $M(\mathrm{~kg})$ & $d(\mathrm{~m})$ & $\zeta$ & $\zeta_{a}$ & $\omega_{n}(\mathrm{rad} / \mathrm{s})$ & $\omega_{\text {na }}(\mathrm{rad} / \mathrm{s})$ & $I\left(\mathrm{kgm}^{2}\right)$ & $F(\mathrm{Nms})$ \\
\hline 1 & Female & 65 & 0.3 & 0.09 & 0.07 & 7.15 & 6.87 & 0.13 & 0.17 \\
\hline 2 & Female & 58 & 0.3 & 0.1 & 0.06 & 7.56 & 7.08 & 0.05 & 0.07 \\
\hline 3 & Female & 63 & 0.31 & 0.07 & 0.06 & 7.35 & 7.09 & 0.18 & 0.19 \\
\hline 4 & Male & 70 & 0.31 & 0.06 & 0.04 & 6.92 & 6.57 & 0.09 & 0.08 \\
\hline 5 & Male & 78 & 0.32 & 0.1 & 0.08 & 7.57 & 7.18 & 0.14 & 0.21 \\
\hline 6 & Female & 70 & 0.3 & 0.08 & 0.06 & 7.98 & 7.23 & 0.09 & 0.11 \\
\hline 7 & Male & 86 & 0.32 & 0.09 & 0.08 & 7.01 & 6.61 & 0.3 & 0.39 \\
\hline 8 & Male & 90 & 0.33 & 0.1 & 0.09 & 7.08 & 6.79 & 0.27 & 0.38 \\
\hline Mean \pm SD & & & & $0.09 \pm 0.02$ & & $7.33 \pm 0.36$ & & $0.16 \pm 0.09$ & \\
\hline
\end{tabular}

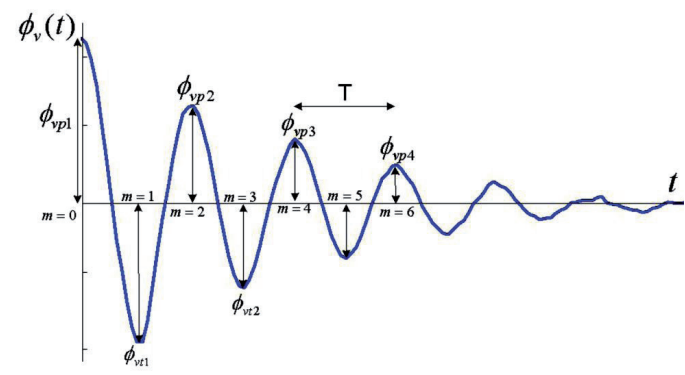

Fig. 9: Typical recording of time against elbow angle during a pendulum test. The time period, $T$, and the amplitude of the first to fourth peaks, $\varphi_{v p 1}$ to $\varphi_{v p 4}$, and the first and second troughs, $\varphi_{v t 1}$ and $\varphi_{v t 2}$, are indicated. The turning points are also indicated using the index $m$.
2) Initial Estimate of the Undamped Natural Frequency of the Elbow Joint, $\omega_{n}$ : The period of the pendulum swing, $T$, is related to the undamped natural frequency of the elbow joint, $\omega_{n}$, and its damping ratio was shown in equation 31:

$$
T=\frac{2 \pi}{\omega_{n} \sqrt{1-\zeta^{2}}}
$$

which can be rearranged to give an expression for $\omega_{n}$ :

$$
\omega_{n}=\frac{2 \pi}{\sqrt{1-\zeta^{2}} T}
$$




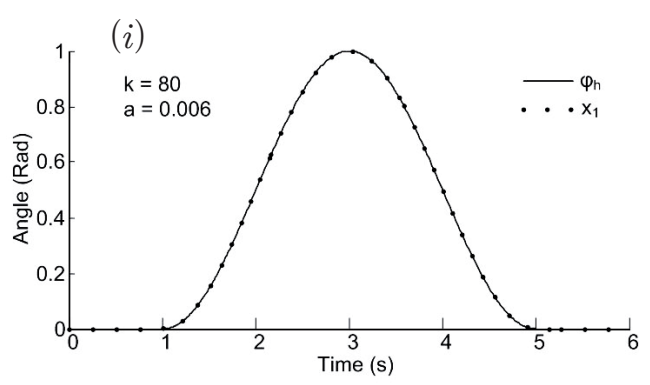

(ii)

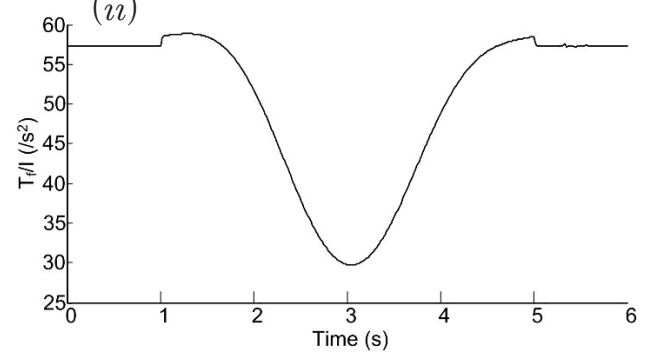

Fig. 10: (i). Joint angle with respect to the horizontal, $\varphi_{h}$, is plotted against time (solid line). The state variable $x_{1}$ is also plotted against time (dotted line), for $k=80$ and $a=0.006$. (ii) The model output, an estimate of $\frac{T_{f}}{I}$ is presented.

Using the equation in this form leads to numerical inaccuracy in calculating $\sqrt{1-\zeta^{2}}$, because we have found $\zeta<<1$. This step is avoided by rearranging the equation for $\omega_{n}$ as:

$$
\omega_{n}=\frac{2 \pi \zeta}{\sqrt{1-\zeta^{2}} T \zeta}
$$

or

$$
\omega_{n}=\frac{\delta}{T \zeta}
$$

An initial estimate of $\omega_{n}$ was calculated, with and without an added weight at the wrist.

3) Optimisation procedure for $\zeta$ and $\omega_{n}$ : An unconstrained nonlinear optimisation was then conducted, using Matlab (The Mathworks). The difference between a measured pendulum curve and the theoretical curve, equation 27, was minimised using a Gaussian least squares error technique. Illustrations for three pendulum tests are shown in Fig. 11.

4) The Moment of Inertia of the Elbow Joint, $I$ : Using equations 3 and 4 , it can be shown that the moment of inertia, $I$, is related to $\zeta$ and $\omega_{n}$ according to the following equation:

$$
2 \zeta \omega_{n}=\frac{F}{I}
$$
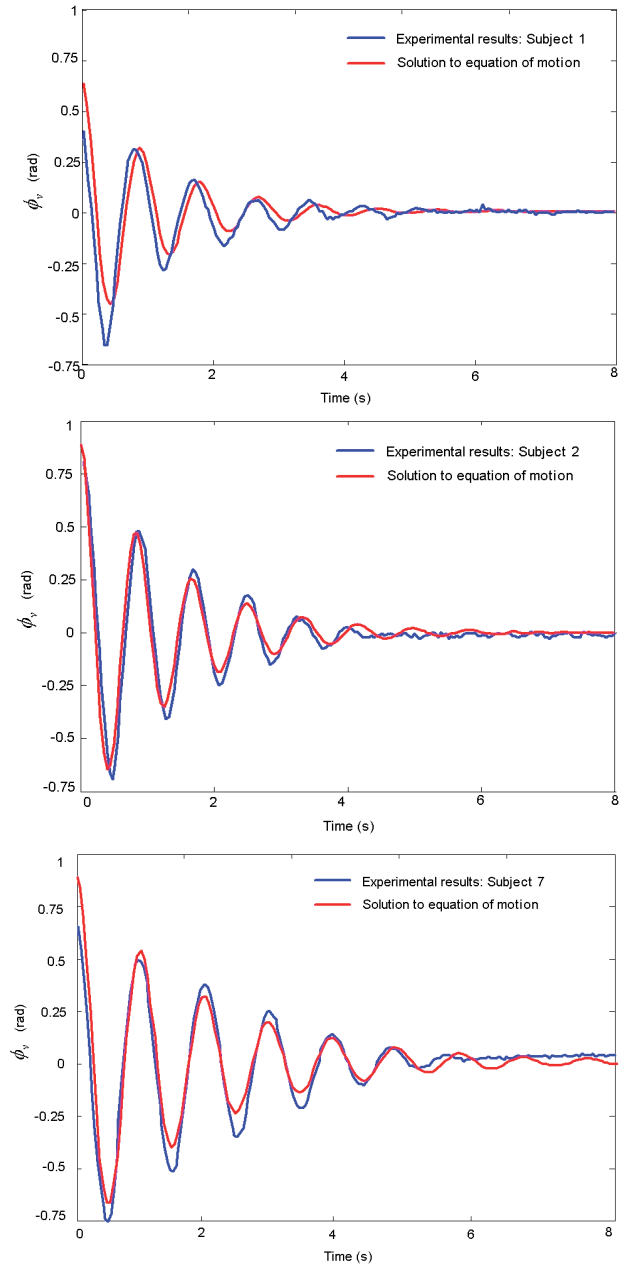

Fig. 11: Three pendulum test recordings compared with the solution to their corresponding equations of motion.

Since $F$ cannot be measured directly using these experiments, a weight was added to the wrist and the pendulum test procedure was repeated, giving:

$2 \zeta_{a} \omega_{n a}=\frac{F}{I+m d^{2}}$

where $\zeta_{a}$ and $\omega_{n a}$ are the values of the damping ratio and the undamped natural frequency of the elbow with an added $0.45 \mathrm{~kg}$ weight at the wrist. I can then be calculated by combining equations 35 and 36, giving:

$$
I=\frac{m d^{2}}{\frac{\zeta \omega_{n}}{\zeta_{a} \omega_{n a}}-1}
$$


I was calculated for each subject. Individual and mean values are given in Table 1.

5) The Friction Coefficient of the Elbow Joint, F: Equation 35 may be manipulated in order to find the friction coefficient, $F$. The computed value of $F$ for each subject is presented in Table 1.

$$
F=2 \zeta \omega_{n} I
$$

\section{Model Results}

A movement trajectory, $\varphi_{v}(t)$, shown on Figure 10(i), was imported into Simulink. The controller was then tuned, using parameter values for subject 5 , so that $\varphi_{v}(t)$ was matched by $x_{1}(t)$ as closely as possible. The values $\mathrm{k}=80$ and $\mathrm{a}=0.006$ gave such a good match that the two graphs could not be distinguished from each other. This indicates that, subject to the accuracy of $\zeta$ and $\omega_{n}$, the graph of y vs. t shown on Figure 10(ii) must also be a close representation of the unmeasurable net elbow torque per unit moment of inertia, $\frac{T_{f}}{I}(t)$.

\section{Discussion}

The model developed in this study may be used to predict net joint torque, which could be used to assess variations in movement patterns due to neuromuscular disease, or variations in biomechanical parameters between different subject groups. This type of model could also be used in the development of a musculoskeletal model or a neuromusculoskeletal model. The accuracy of the model results depends on the accuracy of the values $\zeta, \omega_{n}, a$ and $k$. In order to find unnormalised joint torque, the moment of inertia, $I$, must also be known. The controller was tuned so that reliable values of $a$ and $k$ were obtained, and an experimental procedure was employed to measure $\zeta$ and $\omega_{n}$. Passive muscle force was neglected from the equation of motion of the system, which could have an effect on the results of the pendulum test study. A solution to the pendulum equation of motion was compared with experimentally recorded pendulum tests, using subject specific values of $\zeta$ and $\omega_{n}$ in each case. Three examples of this comparison are presented in Figure 11, showing a good match. Similarly close matches were obtained for other subjects. Therefore, the methods derived in this study to calculate the biomechanical parameters $\zeta, \omega_{n}$ and $I$ were considered to be reliable. The method used in this study to measure the moment of inertia of the elbow joint, using an added mass a known distance from the joint, was developed independently, had been used previously by [12] for the knee joint. However, equation 37 was derived using an alternative and much simpler method in this study than in [12]. Previously, an almost constant value of 0.125 for $\zeta$ was found for the knee joint by [2] and [1]. This is not the case for the elbow joint in our study. The biomechanical parameters of the elbow joint measured in this study have been previously shown to vary significantly with gender [9], and between subjects in a group of male stroke patients, indicating that a subject-specific method should be used to measure these parameters.

\section{Conclusion}

An inverse dynamics model of the elbow joint has been developed, using control theory. A nonlinear observer was designed, which could estimate joint torque per unit moment of inertia, based on a measured movement trajectory. The physiological parameters required by the model were measured using the pendulum test at the elbow joint, and their accuracy was indicated by the goodness of fit obtained between the pendulum swing curves and the analytical solution to the linearised pendulum. The new method presented here allowed the moment of inertia of the elbow joint to be easily measured. The inverse dynamics model developed in this study could be a useful tool for clinicians in analysing kinematics in patients with neuromuscular disorders or who undergone orthopaedic surgery.

\section{References}

[1] T. Bajd and L. Vodovnik. Pendulum testing of spasticity. J Biomed Eng, 6(1):9-16, 1984.

[2] A. de Paor, D. Burke, and C. O'Connor. Application of the earth's magnetic field and accelerometry to the measurement of net knee extensor torque. Measurement Science Review, 1:15-18, 2001.

[3] R. C. Dorf and R. H. Bishop. Modern control systems. Upper Saddle River, NJ: Pearson Education, 2005.

[4] J. W. Jr Fee and F. Miller. The leg drop pendulum test performed under general anesthesia in spastic cerebral palsy. Dev Med Child Neurol, 46(4):273-81, 2004.

[5] H. Hatze. A new method for the simultaneous measurement of the movement of inertia, the damping coefficient and the location of the centre of mass of a body segment in situ. Eur J Appl Physiol Occup Physiol, 34(4):217-26, 1975.

[6] H. J. Hermens, B. Freriks, R. Merletti, D. Stegeman, J. Blok, and 
G. Rau. SENIAM 8: European recommendations for surface electromyography. Enschede: Roessengh Research and Development, 1999.

[7] E. L. Hutchins, R. V. Gonzalez, and R. E. Barr. Comparison of experimental and analytical torque-angle relationships of the human elbow joint complex. Biomed Sci Instrum, 29:17-24, 1993.

[8] R. E. Kearney and I. W. Hunter. System identification of human joint dynamics. Crit Rev Biomed Eng, 18:55-87, 1990.

[9] C. C. Lin, M. S. Ju, and H. W. Huang. Gender and age effects on elbow joint stiffness in healthy subjects. Arch Phys Med Rehabil, 86(1):82-5, 2005.

[10] C. C. Lin, M. S. Ju, and H. W. Huang. Muscle tone in diabetic polyneuropathy evaluated by the quantitative pendulum test. Arch Phys Med Rehabil, 88(3):368-73, 2007.

[11] C. C. Lin, M. S. Ju, and C. W. Lin. The pendulum test for evaluating spasticity of the elbow joint. Archives Phys Med Rehab, 84:69-74, 2003.

[12] D. C. Lin and W. Z. Rymer. A quantitative analysis of pendular motion of the lower leg in spastic human subjects. IEEE Trans Biomed Eng, 38(9):906-18, 1991.

[13] A. O'Dwyer. Handbook of PI and PID controller tuning rules. London: Imperial College Press, 2003.

[14] Q. Peng, P. Shah, R. W. Selles, D. J. Gaebler-Spira, and L. Q. Zhang. Measurement of ankle spasticity in children with cerebral palsy using a manual spasticity evaluator. Conf Proc IEEE Eng Med Biol Soc, 7:4896-9, 2004.

[15] R. Riener and A. Straube. Inverse dynamics as a tool for motion analysis: arm tracking movements in cerebellar patients. J Neurosci Methods, 72(1):87-96, 1997.

[16] G. Shue, P. E. Crago, and H. J. Chizeck. Muscle-joint models incorporating activation dynamics, moment-angle, and moment-velocity properties. IEEE Trans Biomed Eng, 42:212-223, 1995.

[17] R. B. Stein, E. P. Zehr, M. K. Lebiedowska, D. B. Popovic, A. Scheiner, and H. J. Chizeck. Estimating mechanical parameters of leg segments in individuals with and without physical disabilities. IEEE Trans Rehabil Eng, 4(3):201-11, 1996.

[18] R. Wartenburg. Pendulousness of the legs as a diagnostic test. J. Biomed Eng, 1:18-24, 1951. 